\title{
Diffuse Idiopathic Pulmonary Neuroendocrine Cell Hyperplasia: A Clinical Case
}

\author{
Adelaide Pereira Alves, Ana Barroso, Margarida Dias \\ Centro Hospitalar de Vila Nova de Gaia/Espinho, Vila Nova de Gaia, Portugal
}

Received: 10/12/2019

Accepted: 31/12/2019

Published: $17 / 02 / 2020$

How to cite this article: Pereira Alves A, Barroso A, Dias M. Diffuse idiopathic pulmonary neuroendocrine cell hyperplasia: a clinical case. EJCRIM 2020;7: doi:10.12890/2020_001422.

Conflicts of Interests: The Authors declare that there are no competing interest

This article is licensed under a Commons Attribution Non-Commercial 4.0 License

\section{ABSTRACT}

Diffuse idiopathic pulmonary neuroendocrine cell hyperplasia (DIPNECH) is a rare pulmonary condition characterized by diffuse proliferation of neuroendocrine cells in the epithelium of the bronchial wall. DIPNECH may be easily missed in daily clinical practice and diagnosis is often delayed, which may impair prognosis since this condition is considered a pre-invasive lesion for lung carcinoid tumours. We report a clinical case of DIPNECH in order to discuss the diagnostic and therapeutic approach for this entity, the management of which is not yet well established in the literature.

\section{LEARNING POINTS}

- DIPNECH is a poorly understood lung condition characterized histologically by diffuse proliferation of pulmonary neuroendocrine cells in the bronchial wall, clinically by obstructive respiratory symptoms and radiologically by small airway disease features and pulmonary nodules.

- $\quad$ DIPNECH is considered to be a preneoplastic lesion within the spectrum of pulmonary neuroendocrine tumours.

- Treatment is usually guided by the symptoms and prognosis is highly variable.

\section{KEYWORDS}

Diffuse idiopathic pulmonary neuroendocrine cell hyperplasia (DIPNECH), pulmonary neuroendocrine tumours

\section{INTRODUCTION}

Diffuse idiopathic pulmonary neuroendocrine cell hyperplasia (DIPNECH) is a rare lung condition characterized histologically by a diffuse proliferation of neuroendocrine cells (NECs) within bronchial mucosal epithelium without basement membrane breakthrough ${ }^{[1]}$. If basement membrane breakthrough occurs, the lesion is called a carcinoid tumourlet if the nodule diameter is $\leq 5 \mathrm{~mm}$ and a carcinoid tumour if the nodule diameter is $>5 \mathrm{~mm}^{[2]}$. Information regarding the natural history and management of DIPNECH is scarce; this can be troublesome in clinical practice since DIPNECH is an under-recognized entity, which is considered to be a pre-invasive lung lesion within the spectrum of NEC neoplasia, according to the World Health Organization ${ }^{[3]}$.

\section{CASE DESCRIPTION}

A 61-year-old female was referred to our pulmonology outpatient clinic because of dry cough and progressive dyspnoea for approximately 1 year. She had no other respiratory symptoms, no orthopnoea or paroxysmal nocturnal dyspnoea, no gastro-oesophageal reflux symptoms, no nasal complaints and no constitutional symptoms. She was a never smoker who previously worked as a gardener. She had a past medical history of arterial hypertension, type 2 diabetes mellitus, dyslipidaemia and obesity grade II. She was medicated with lisinopril/ 
hydrochlorothiazide 20/12.5 mg once/day, sitagliptin/metformin 80/850 mg once/day, INN-insulin glargine $48 \cup$ and atorvastatin 20 mg once/day. On physical examination, the patient was afebrile and haemodynamically stable. She had no signs of respiratory distress and an oxygen saturation of $97 \%$ at room air. Cardiac auscultation was normal but pulmonary auscultation revealed diffuse wheezing. No peripheral oedemas or other relevant changes were found.

Diagnostic investigation began with a chest x-ray, which was normal, and a pulmonary function test showing a mild obstructive ventilatory defect with a negative bronchodilator response and a diffusing capacity of the lungs for carbon monoxide (DLCO) of 5.08 $\mathrm{mm} / \mathrm{min} / \mathrm{kPa}$ (78\% of the expected value). The patient was treated with an inhaled bronchodilator and corticosteroid, without significant clinical improvement. Diagnostic work-up continued with chest computed tomography (CT) revealing multiple pulmonary nodules: 3 in the left lower lobe (LLL) of $16 \mathrm{~mm}, 5 \mathrm{~mm}$ and $2 \mathrm{~mm}$; 2 in the right lower lobe (RLL) of $7 \mathrm{~mm}$ and $9 \mathrm{~mm} ; 2$ in the right upper lobe of $3 \mathrm{~mm}$ and $2 \mathrm{~mm}$ and 1 in the left upper lobe of $3 \mathrm{~mm}$. CT-guided transthoracic needle biopsies (TNBs) of the 2 largest nodules were performed. Fig. 1 shows the 2 biopsied pulmonary nodules on the chest CT scans. TNB of the $16 \mathrm{~mm}$ LLL nodule revealed a typical carcinoid tumour $\left(0 \mathrm{mitoses} / 2 \mathrm{~mm}{ }^{2}, \mathrm{no}\right.$ necrosis, Ki-67: <5\%). TNB of the $9 \mathrm{~mm}$ RLL nodule suggested a neuroendocrine lesion (typical carcinoid tumour? Tumourlet?) without visible mitosis. The patient also underwent flexible bronchoscopy which showed no endobronchial lesions. Positron emission tomography/CT using Ga-68labelled somatostatin analogues (Ga-68 DOTANOC PET/CT) showed no expression of somatostatin receptors. No other lesions were found during tumour staging, namely, in abdominal CT and brain magnetic resonance imaging.
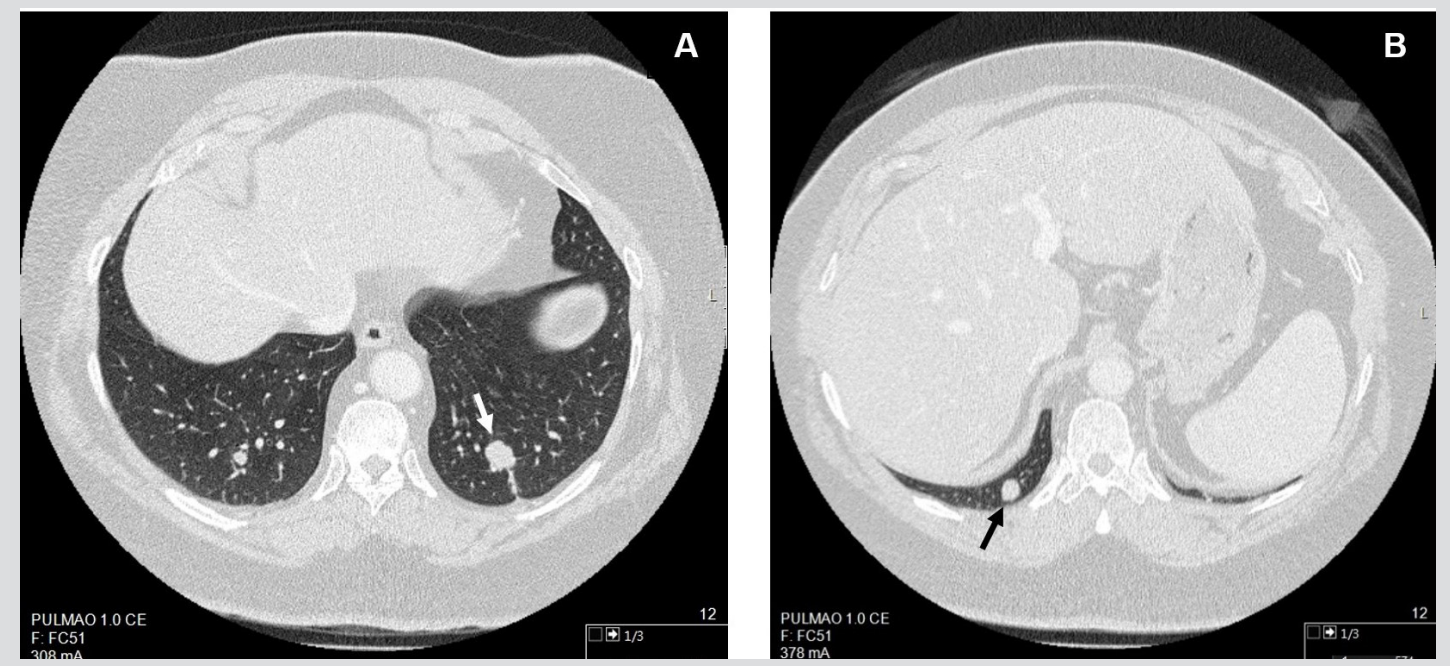

Figure 1. Chest computed tomography

(CT) revealing the 2 pulmonary nodules (indicated by arrows) of which CT-guided transthoracic needle biopsies were performed: $\mathrm{A}-16 \mathrm{~mm}$ nodule in the left lower lobe; $B-9 \mathrm{~mm}$ nodule in the right lower lobe

A diagnosis of a typical carcinoid tumour (cT1bNOMO) of the LLL plus a possible synchronous typical carcinoid tumour (cT1aNOM0) of the RLL was assumed. The case was discussed at the multidisciplinary thoracic tumour meeting of our hospital centre and it was decided to treat the patient with left inferior lobectomy and right inferior lobectomy. These procedures were performed at the same surgical time through bilateral uniportal video-assisted thoracic surgery resection, without complications. The patient underwent pulmonary rehabilitation prior to and after surgery.

Regarding the histology of the surgical specimens, 1 nodule of $1.6 \times 1.6 \times 1.3 \mathrm{~cm}$ was found in the LLL and 3 nodules were found in the RLL, the largest in size being $1.5 \times 1 \times 0.7 \mathrm{~cm}$. All nodules were morphologically similar and all of them presented $<2 \mathrm{mitoses} / 2 \mathrm{~mm}{ }^{2}$, no necrosis, Ki-67 expression of $5 \%$ and were positive for CD56, chromogranin A and synaptophysin. No pleural invasion was found and the bronchial stump was tumour-free. Interestingly, several tumourlets and NEC hyperplasia were found in the remaining lung parenchyma. Tumour positivity was also found in the left hilar and $9 \mathrm{~L}$ lymph nodes.

After analyzing the surgical specimens, we established the final diagnosis of synchronous bronchial typical carcinoid tumours plus several tumourlets and DIPNECH. It was decided by the multidisciplinary medical team that the patient had no criteria for adjuvant therapy. Currently, the patient has been under surveillance for 1 year without evidence of relapse. 


\section{DISCUSSION}

Through this clinical case, we intend to spread awareness of DIPNECH and its typical clinical presentation. Just as in the presented case, DIPNECH usually affects non-smoker females in the sixth decade of life and the most common symptoms are chronic shortness of breath, dry cough and wheezing. In some cases, however, DIPNECH may be an incidental diagnosis following chest CT or from a surgical specimen ${ }^{[2,4]}$. Pulmonary function tests typically reveal an obstructive defect. A possible explication is the fact that pulmonary NECs are mainly found in the intrapulmonary airways, especially at bifurcations. In addition, it has been hypothesized that these cells secrete bombesin-like peptides which have bronchoconstrictive effects. These facts may explain not only the obstructive pattern in pulmonary function tests, but also, the respiratory symptoms, mimicking asthma. For these reasons, treatment with a short course of inhaled steroids to reduce inflammation and bronchoconstriction has been carried out in clinical practice ${ }^{[2,4]}$.

The localization of NECS around small airways may also explain radiological manifestations of DIPNECH, typically including mosaic attenuation due to air-trapping, bronchial wall thickening and the presence of small pulmonary nodules ${ }^{[2]}$. In this clinical case, there was no radiological evidence of small airways disease but the patient had several small pulmonary nodules. In addition to neuroendocrine tumours, DIPNECH has also been associated with lung adenocarcinom $a^{[5]}$. Being aware of this association is particularly important, since the DIPNECH imaging findings may be misdiagnosed as metastatic disease, which may have relevant implications with respect to treatment decisions.

Definitive diagnosis of DIPNECH requires biopsy and an adequately large tissue sample must be obtained to identify any associated lesions $^{[2]}$. In this case, it was possible to identify within the surgical specimens DIPNECH, carcinoid tumourlet and carcinoid tumour presence, reinforcing that these may actually represent different histological manifestations within the range of neuroendocrine lesions, with a continuity and correlation.

Treatment is not well established and should be guided by symptoms. If the patient is asymptomatic, maintained surveillance of the development of symptoms and the carcinoid tumour with chest CT every 12-24 months is recommended. If symptoms are present, treatment options include surgery (pulmonary resection or lobectomy), pharmacological treatment (namely, with somatostatin analogues and inhaled steroids) and in very severe cases, lung transplantation ${ }^{[4]}$.

The prognosis of DIPNECH is highly variable and seems to correlate better with the severity of obstructive respiratory symptoms than with the development of the carcinoid tumour. Most patients have stable or slowly progressive disease. Some patients, however, may progress to severe airway obstruction and respiratory failure ${ }^{[2]}$.

\section{REFERENCES}

1. Wirtschafter E, Walts AE, Liu ST, Marchevsky AM. Diffuse idiopathic pulmonary neuroendocrine cell hyperplasia of the lung (DIPNECH): current best evidence. Lung 2015;193:659-667.

2. Rossi G, Cavazza A, Spagnolo P, Sverzellati N, Longo L, Jukna A, et al. Diffuse idiopathic pulmonary neuroendocrine cell hyperplasia syndrome. Eur Respir J 2016;47(6):18291841.

3. Travis WD, Brambilla E, Burke AP, Marx A, Nicholson AG. WHO classification of tumors of the lung, pleura, thymus and heart. 4th ed. Lyon: IARC Press; 2015 , p. 78-79.

4. Myint ZW, McCormick J, Chauhan A, Behrens W, Anthony LB. Management of diffuse idiopathic pulmonary neuroendocrine cell hyperplasia: review and a single center experience. Lung 2018;196(5):577-581.

5. Gorospe L, Muñoz-Molina GM, Farfán-Leal FE, Cabañero-Sánchez A, García-Gómez-Muriel I, Benito-Berliches A. Association of diffuse idiopathic pulmonary neuroendocrine hyperplasia (DIPNECH) with lung adenocarcinoma: a radiologist`s perspective. Lung Cancer 2017;110:77-79. 